\title{
Activation of Phox2b-Expressing Neurons in the Nucleus Tractus Solitarii Drives Breathing in Mice
}

\author{
[CCongrui Fu, ${ }^{1,2 \star}$ Luo Shi, ${ }^{1 \star}$ Ziqian Wei, ${ }^{1}$ Hongxiao Yu, ${ }^{1}$ Yinchao Hao, ${ }^{1}$ Yanming Tian, ${ }^{1}$ Yixian Liu, ${ }^{1}$ Yi Zhang, ${ }^{1}$ \\ Xiangjian Zhang, ${ }^{3}$ Fang Yuan, ${ }^{1}$ and ${ }^{-S h e n g ~ W a n g ~}{ }^{1,4}$

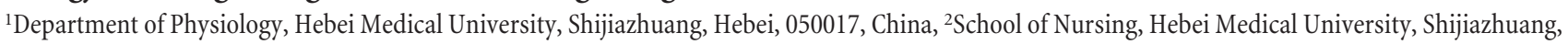 \\ Hebei, 050000, China, ${ }^{3} \mathrm{Hebei}$ Key Laboratory of Vascular Homeostasis and Hebei Collaborative Innovation Center for Cardio-cerebrovascular Disease, \\ Shijiazhuang, Hebei, 050000, China, and ${ }^{4}$ Neuroscience Center, Hebei Medical University, Shijiazhuang, Hebei, 050017, China
}

The nucleus tractus solitarii (NTS) is implicated in the control of breathing, but the neuronal phenotype and circuit mechanism involved in such a physiological function remain incompletely understood. This study focused on the respiratory role of paired-like homeobox $2 b$ gene (Phox 2b)-expressing NTS neurons and sought to determine whether selective stimulation of this set of neurons activates breathing in male mice. A Cre-dependent vector encoding a Gq-coupled human M3 muscarinic receptor (hM3Dq) was microinjected into the NTS of Phox2b-Cre transgenic mice. The hM3Dq-transduced neurons were pharmacologically activated in conscious mice while respiratory effects were measured by plethysmography. We demonstrate that chemogenetic stimulation of Phox $2 b$-expressing NTS neurons significantly increased baseline minute volume via an increase in respiratory frequency rather than tidal volume. Chemogenetic stimulation also synergized with moderate $\mathrm{CO}_{2}$ stimulation to enhance pulmonary ventilatory response. Selective ablation of Phox $2 \mathrm{~b}$-expressing NTS neurons notably attenuated a hypercapnic ventilatory response. Moreover, histological evidence revealed that stimulation of Phox $2 b-$ expressing NTS neurons increased neuronal activity of the preBötzinger complex. Finally, we presented the neuroanatomical evidence of direct projection of Phox $2 b$-expressing NTS neurons to putative respiratory central pattern generator. Overall, these findings suggest that selective activation of Phox $2 \mathrm{~b}$-expressing NTS neurons potentiates baseline pulmonary ventilation via an excitatory drive to respiratory central pattern generator and this group of neurons is also required for the hypercapnic ventilatory response.

Key words: breathing; chemogenetics; chemoreceptor; nucleus tractus solitarii; Phox2b

\section{Significance Statement}

The nucleus tractus solitarii (NTS) has been implicated in the control of breathing. The paired-like homeobox $2 b$ gene (Phox $2 b)$ is the disease-defining gene for congenital central hypoventilation syndrome and is restrictively present in brainstem nucleus, including the NTS. Using a chemogenetic approach, we demonstrate herein that selective stimulation of Phox2b-expressing NTS neurons vigorously potentiates baseline pulmonary ventilation via an excitatory drive to respiratory central pattern generator in rodents. Genetic ablation of these neurons attenuates the hypercapnic ventilatory response. We also suggest that a fraction of Phox2b-expressing neurons exhibit $\mathrm{CO}_{2}$ sensitivity and presumably function as central respiratory chemoreceptors. The methodology is expected to provide a future applicability to the patients with sleep-related hypoventilation or apnea.

\section{Introduction}

Congenital central hypoventilation syndrome (CCHS) is a rare autosomal dominant disease defined by a failure in autonomic

Received Aug. 11, 2018; revised Dec. 17, 2018; accepted Dec. 28, 2018.

Author contributions: Y.Z., X.Z., and F.Y. edited the paper; S.W. wrote the first draft of the paper; C.F., F.Y., and S.W. designed research; C.F., L.S., Z.W., H.Y., Y.H., Y.L., and F.Y. performed research; C.F., L.S., H.Y., Y.T., F.Y., and S.W. analyzed data; S.W. wrote the paper.

This work was supported by National Natural Science Foundation of China Grants 31371166 and 31571174 to S.W., Hebei Natural Science Foundation Grant (2014206303, and Hebei Province Government Grant CXZZBS2017102 to C.F. We thank Professor Sergey Kasparov for his comments on an earlier version of the manuscript.

The authors declare no competing financial interests. control of breathing. CCHS patients characteristically exhibit alveolar hypoventilation with diminutive tidal volume (TV) and respiratory rate more frequently during sleep (Weese-Mayer et al., 2010). The paired-like homeobox $2 \mathrm{~b}$ gene (Phox $2 \mathrm{~b}$ ) is the disease-defining gene for CCHS, and the mutation of this gene in animal models results in hypoventilation; the Phox $2 \mathrm{~b}$ gene encodes a transcription factor that plays a critical role in the devel-

\section{*C.F. and L.S. contributed equally to this work.}

Correspondence should be addressed to Sheng Wang at wangsheng@hebmu.edu.cn or Fang Yuan at yuanfphy@163.com.

https://doi.org/10.1523/JNEUROSCI.2048-18.2018

Copyright $\odot 2019$ the authors 
A

AAV2-EF1a-DIO-hM3Dq-mCherry EF1a К«љчวu-baEWप WPRE-pA

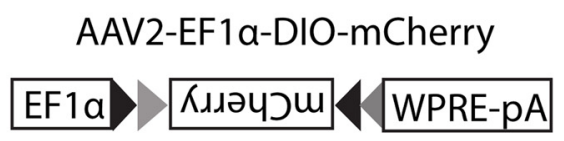

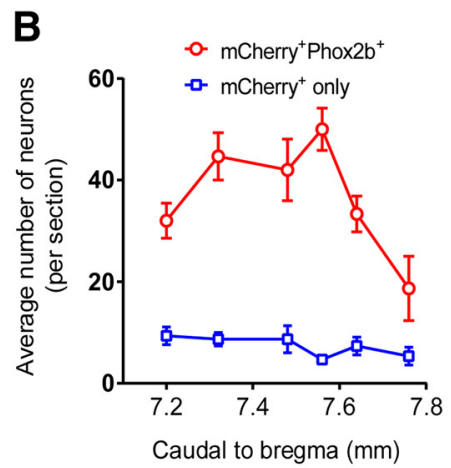

D

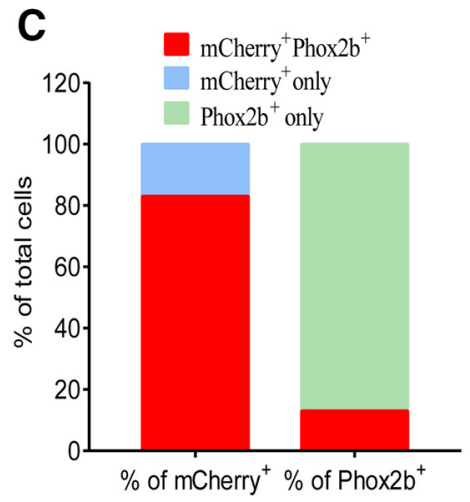

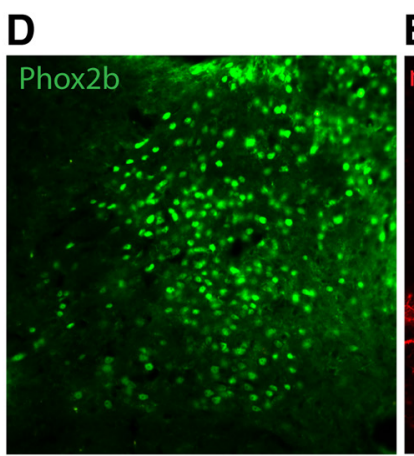

E

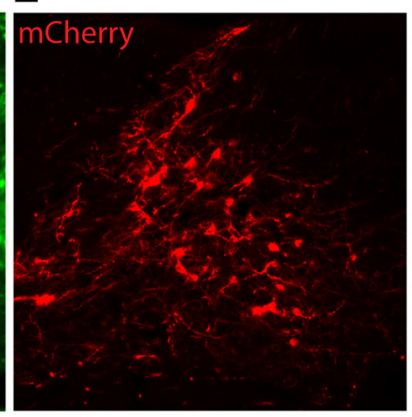

$\mathbf{F}$

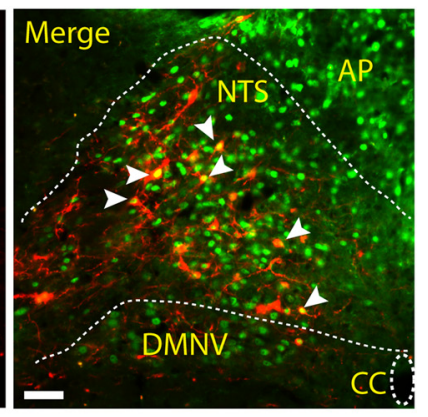

Figure 1. Validation of viral transfection in a Phox2b-Cre mouse line. A, Stereotaxic delivery of AAVs encoding Cre-dependent hM3Dq-mCherry or mCherry into the NTS in Phox2b-Cre mice. $B$, Rostrocaudal distribution of $\mathrm{mCherry}{ }^{+}$Phox $2 \mathrm{~b}^{+}$and $\mathrm{mCherry}{ }^{+} \mathrm{NTS}$ neurons. Cell counts were obtained in 6 coronary sections $(25 \mu \mathrm{m})$ from each mouse $(n=6)$. $C$, Quantification of the percentage of $\mathrm{mCherry}^{+}$Phox $2 \mathrm{~b}^{+}$neurons based on cell counts shown in $\boldsymbol{B}$. The $\mathrm{mCherry}{ }^{+}$Phox $2 \mathrm{~b}^{+}$neurons accounted for $\sim 83 \%$ of total number of $\mathrm{mCherry}{ }^{+}$-expressing neurons and for $\sim 13 \%$ of Phox $2 b^{+}$-expressing neurons. $\mathbf{D}-\boldsymbol{F}$, Most $\mathrm{m}$ Cherry-transduced cells are Phox2b-positive. The merged photomicrograph was derived from Phox $2 \mathrm{~b}$ immunoreactivity (green, $\boldsymbol{D}$ ) and $\mathrm{mCherry}($ red, $\boldsymbol{E}$ ). White arrows indicate the overlap of Phox $2 \mathrm{~b}$ and $\mathrm{mCherry}$. Scale bar, $25 \mu \mathrm{m}$.

opment of autonomic nervous system (Pattyn et al., 1999; Dauger et al., 2003; Tiveron et al., 2003). Phox $2 \mathrm{~b}$ is restrictively present in the afferent pathways of autonomic reflexes, including the nucleus tractus solitarii (NTS) and retrotrapezoid nucleus (RTN), all these regions presumed to contain respiratory chemoreceptors, whereas Phox $2 \mathrm{~b}$ is absent in the respiratory central pattern generator (rCPG) (Dubreuil et al., 2009a). The contribution of Phox2b-expressing NTS neurons to breathing control is incompletely understood.

The NTS, located in the dorsal medulla, integrates the afferent information from both respiratory and cardiovascular receptors (Guyenet, 2006; Dean and Putnam, 2010) and therefore plays a key role in cardiorespiratory regulation. Although several lines of evidence have demonstrated the role of NTS in the control of breathing, very little is known regarding the neurochemical phenotype of respiration-related NTS neurons. Recently, the respiratory role of Phox 2b-expressing medullary neurons has been intensively studied. RTN neurons failed to develop in transgenic mice with mutated form of Phox $2 \mathrm{~b}$, and these mice displayed impaired hypercapnic ventilatory responses, in favor of a crucial role of RTN neurons in the control of breathing (Dubreuil et al., 2009b). Optogenetic stimulation of Phox2b-expressing RTN neurons could activate breathing (Abbott et al., 2009; Kanbar et al., 2010); by contrast, lesions of this set of neurons produced an attenuation of the central respiratory chemoreflex (Takakura et al., 2008). In addition, the majority of Phox $2 \mathrm{~b}$-expressing RTN neurons exhibited an intrinsic $\mathrm{pH}$ sensitivity (Lazarenko et al., 2009; Wang et al., 2013a) that was primarily mediated by G protein-coupled receptor 4 and TASK-2 channels (Wang et al., 2013b; Kumar et al., 2015). More recently, we found that chronic lesions of Phox2b-expressing NTS neurons had no effect on resting pulmonary ventilation but considerably impaired the central respiratory chemoreflex; furthermore, a subset of Phox $2 b-$ expressing neurons also manifested an intrinsic $\mathrm{pH}$ sensitivity (Fu et al., 2017). Nevertheless, these findings are not sufficient to conclude that activation of Phox $2 \mathrm{~b}$-expressing NTS neurons modulates baseline pulmonary ventilation. More importantly, lack of circuit mechanism hinders our understanding of whether Phox2b-expressing NTS neurons modulate breathing via an excitatory drive to $\mathrm{rCPG}$.

Here, we used chemogenetics, genetic apoptosis, immunohistochemistry, and plethysmography to determine whether stimulation of Phox $2 \mathrm{~b}$-expressing NTS neurons activated breathing, and whether these neurons were required for a hypercapnic ventilatory response in a Phox $2 \mathrm{~b}$-Cre transgenic mouse line. We found that chemogenetic stimulation of Phox $2 \mathrm{~b}$-expressing neurons vigorously increased baseline pulmonary ventilation. In contrast, selective ablation of these neurons had no effect on baseline ventilation but remarkably attenuated the hypercapnic ventilatory response.

\section{Materials and Methods}

Animals. All experiments were conducted using a bacterial artificial chromosome Phox2b-Cre transgenic mouse line (IMSR catalog \#JAX: 016223, RRID:IMSR_JAX:016223) that was previously generated (Scott et al., 2011). Mice were housed under controlled temperature $\left(21 \pm 1^{\circ} \mathrm{C}\right)$

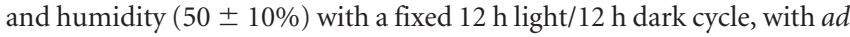
libitum access to food and water. Mice were all on a C57BL/6 genetic background and $\sim 8$ weeks old at the beginning of the experiments. Male mice were used in all experiments. The animal was used in accordance 

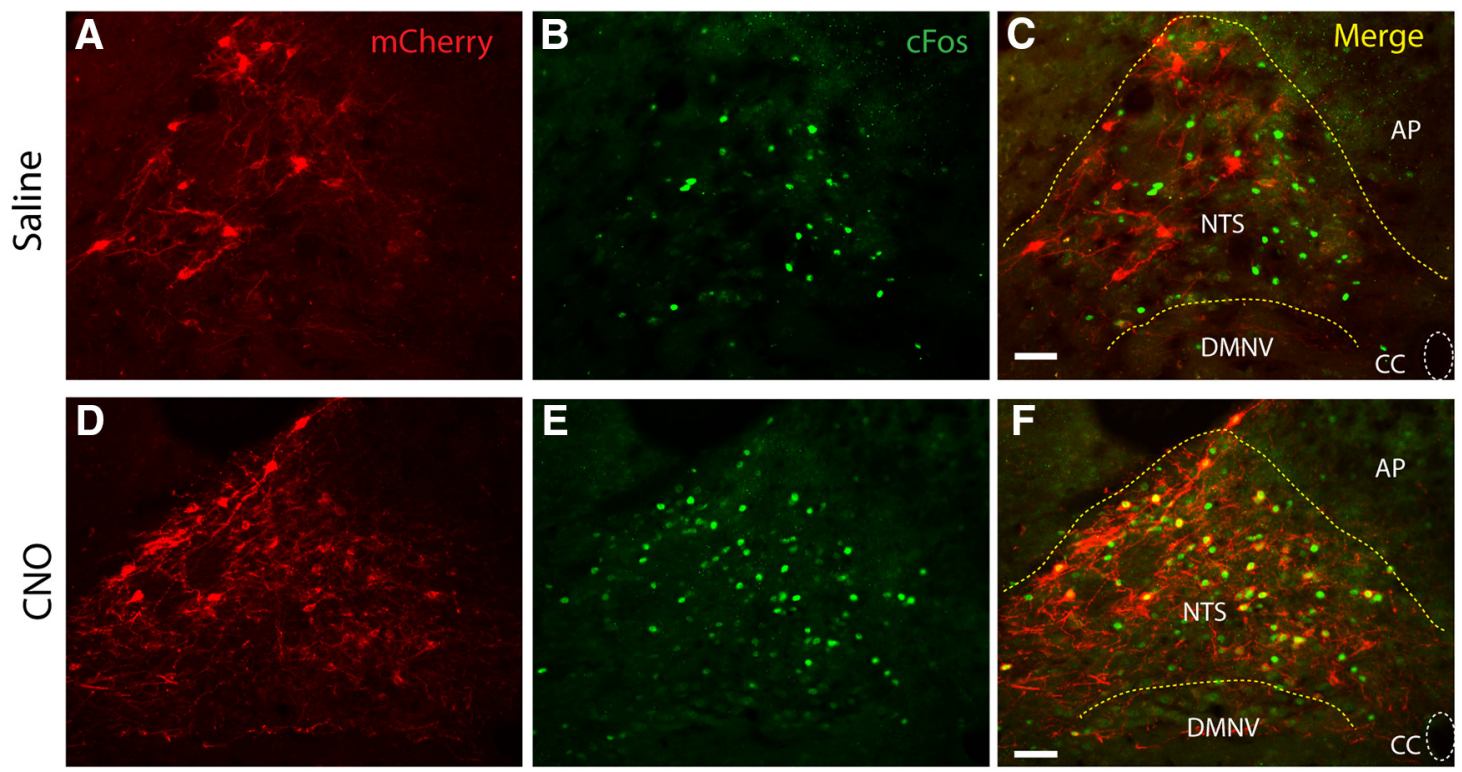

\section{G}

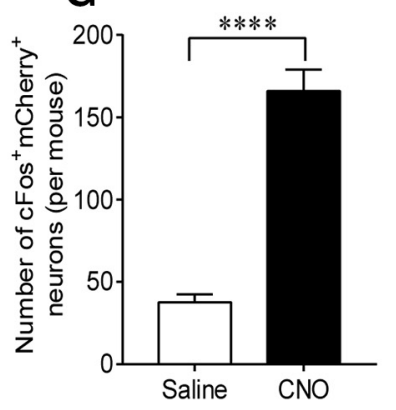

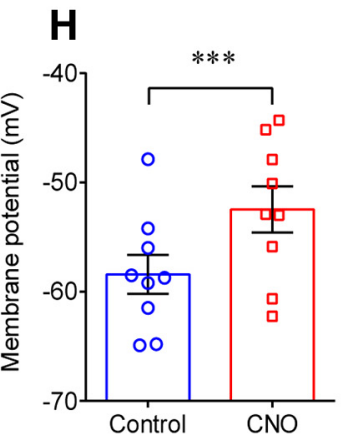

I

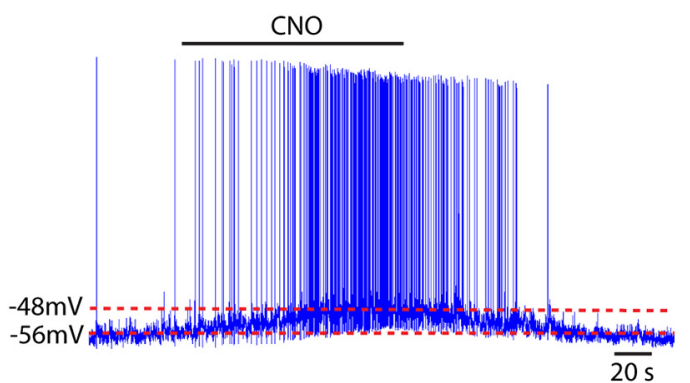

Figure 2. Histomolecular and electrophysiological validation of hM3Dq transduction. $\boldsymbol{A}-\boldsymbol{F}$, Photomicrographs were acquired from Phox2b-Cre mice injected with the AAV2-EF1 $\alpha$-DI0-hM3Dq$\mathrm{mCherry} \mathrm{vector.} \mathrm{Expression} \mathrm{of} \mathrm{mCherry}(\mathrm{red}, \boldsymbol{A}, \boldsymbol{D})$ and $\mathrm{CFos}$ ( $(\mathrm{rreen}, \boldsymbol{B}, \boldsymbol{E})$ was visualized in the NTS region. The merged photomicrograph showed that administration of $\mathrm{CNO}(1 \mathrm{mg} / \mathrm{kg}, \boldsymbol{F})$ activated much more mCherry-transduced neurons compared with saline (C). Immunoreactivity for cFos indicated CNO-activated neurons. Scale bar, $25 \mu \mathrm{m}$. $\mathbf{G}$, The number of cFos ${ }^{+} \mathrm{mCherry}{ }^{+}$neurons after CNO stimulation was greater compared with saline. Cell counts were acquired in 6 coronary sections $(25 \mu \mathrm{m})$ from each Phox $2 \mathrm{~b}$-Cre mouse $\left(n=5\right.$ for each group). ${ }^{* * * *} p<0.0001$ (unpaired $t$ test). $\boldsymbol{H}$, Whole-cell patch-clamp data showing a depolarization after bath application of CNO $(30 \mu \mathrm{m})$ in hM3Dq-transduced neurons $(n=9)$ in acute slices from Phox2b-Cre mice $(n=8)$. ${ }^{* * *} p=0.0003$ (two-tailed paired $t$ test). I, Typical traces illustrating a membrane depolarization $(\sim 6 \mathrm{mV})$ and an increase in firing rate when challenged by bath application of CNO. DMNV, Dorsal motor nucleus of vagus; $\mathrm{AP}$, area postrema; $\mathrm{CC}$, central canal.

with Guide for the care and use of laboratory animals, and was approved by Animal Care and Ethical Committee of Hebei Medical University.

Cre-dependent adeno-associated viral vectors with serotype 2 (AAV2) and their delivery. The AAV2 (titer: $10^{12}$ virus molecules per milliliter) was obtained from GeneChem. The vectors are characterized by an enhanced version of the Gq-coupled human M3 muscarinic receptor (hM3Dq), a type of Designer Receptors Exclusively Activated by Designer Drugs, fused with a mCherry tag under the control of the elongation factor $1 \alpha(\mathrm{EF} 1 \alpha)$ promoter (AAV-EF1 $\alpha$-DIO-hM3Dq-mCherry). The hM3Dq-mCherry sequence is flanked by double lox sites with an inverted reading frame that requires Cre-mediated recombination and inversion into the sense direction for functional expression. At the beginning, the AAV2-EF1 $\alpha$-DIO-mCherry (titer: $10^{12} / \mathrm{ml}$ ) was used to verify the specificity of Cre recombinase expression in Phox2b-expressing neurons.

Phox 2b-Cre mice were subjected to bilateral microinjections of the virus into the NTS. The protocol for injections has been depicted previously (Fu et al., 2017). Shortly, the mice were anesthetized with pentobarbital sodium $(60 \mu \mathrm{g} / \mathrm{g}$, i.p.). Depth of anesthesia was evaluated by an absence of corneal and hindpaw withdrawal reflexes. Additional anesthetic was administered as necessary (30\% of the original dose, i.p.). All surgical procedures were performed under strict aseptic conditions. After anesthesia, the mouse was placed prone on a stereotaxic apparatus (Stoelting), and body temperature was maintained at $37^{\circ} \mathrm{C}$ using a heat- ing pad and a blanket (RWD). The occipital craniotomy was performed to expose dorsal surface over the NTS. A glass micropipette $(25 \mu \mathrm{m})$ filled with the viral vector was microinjected into the dorsal and intermediate NTS at the level of calamus scriptorius (approximate stereotaxic coordinates: mediolateral $\pm 0.3 \mathrm{~mm}$, anteroposterior $0.2 \mathrm{~mm}$, dorsoventral $-0.2 \mathrm{~mm}$; mediolateral $\pm 0.4 \mathrm{~mm}$, anteroposterior $0.3 \mathrm{~mm}$, dorsoventral $-0.3 \mathrm{~mm}$ ) using a syringe pump (Harvard Apparatus). A total volume of $400 \mathrm{nl}$ (100 nl per injection) was injected into the NTS. After injection, the mice received injections of antibiotic ampicillin $(125 \mathrm{mg} /$ $\mathrm{kg}$, i.p.) and the analgesic ketorolac ( $4 \mathrm{mg} / \mathrm{kg}$, i.p.). Mice were then given a 4 week recovery before breathing measurements. The surgical procedures and virus injections produced no observable behavioral effects, and these mice gained weight normally.

Chemogenetic stimulation of the NTS. The ventilatory response was measured in conscious, freely moving mice using whole-body plethysmography (EMKA Technologies) as described previously (Kumar et al., 2015; Fu et al., 2017). Briefly, mice were placed in the chamber ahead of the testing protocol for at least $2 \mathrm{~h}$ (for acclimation). A mass flow regulator provided quiet, constant, and smooth air mixture through the animal chamber $(0.5 \mathrm{~L} / \mathrm{min})$. Mice were exposed to $100 \% \mathrm{O}_{2}$ throughout the whole experiments to inactivate peripheral respiratory chemoreceptors. Breathing parameters, comprising respiratory frequency (RF, breaths/ $\mathrm{min})$, TV $(\mu \mathrm{l} / \mathrm{g})$, minute volume $(\mathrm{MV}, \mu \mathrm{l} / \mathrm{min} / \mathrm{g})$, inspiration time $\left(\mathrm{T}_{\mathrm{i}}\right)$, and expiration time $\left(\mathrm{T}_{\mathrm{e}}\right)$, were measured during behavioral quiescence 

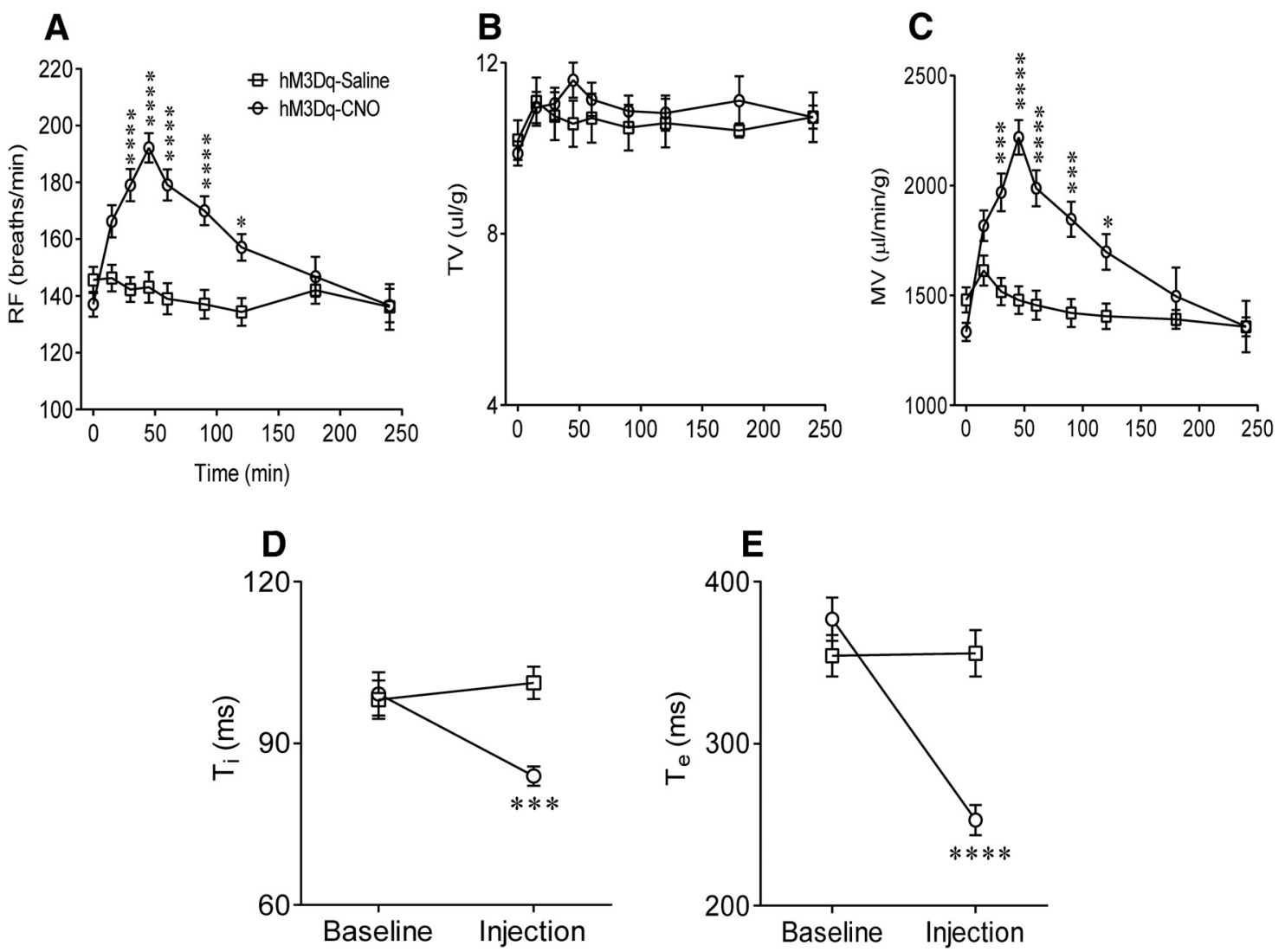

Figure 3. Potentiation of respiratory activity by activation of Phox2b-expressing neurons. In conscious hM3Dq-transduced Phox2b-Cre mice, injections of CNO (1 mg/kg, i.p.) produced greater increases in $\operatorname{RF}(\boldsymbol{A})$ and $\operatorname{MV}(\boldsymbol{C})$ relative to saline injections. Such an increase commenced 15 min after the $C N O$ injection, peaked during $30-60$ min, then fully returned to baseline level at $\sim 180$ min. No significant change in $\operatorname{TV}(\boldsymbol{B})$ was found between two groups. Both $\mathrm{T}_{\mathrm{i}}(\boldsymbol{D})$ and $\mathrm{T}_{\mathrm{e}}(\boldsymbol{E})$ were significantly reduced $45 \mathrm{~min}$ after injection of CN0 compared with saline injection. Numbers of mice: $n=$ 32 for the CNO group; $n=22$ for the saline group. CNO versus saline (two-way ANOVA with Bonferroni's test): ${ }^{*} p<0.05 ;{ }^{* * *} p<0.001 ;{ }^{* * * *} p<0.0001$.

and regular breathing. MV was calculated as the product of the RF and $\mathrm{TV}$, normalized to body weight (grams). For analysis of the acute hypercapnic ventilatory response, we sampled 10 consecutive epochs $(200 \mathrm{~s}$, representing $\sim 500$ breaths at rest) that showed the least interbreath irregularity during the plateau period. Baseline ventilation was initially determined before injection of the activator of hM3Dq, clozapine $\mathrm{N}$-oxide (CNO, $1 \mathrm{mg} / \mathrm{kg}$, dissolved in saline, i.p.), followed by consecutive measurements for $4 \mathrm{~h}$ to assess the stimulatory effect of CNO. In some instances, to examine the additive effect of $\mathrm{CO}_{2}$ and $\mathrm{CNO}$, a hypercapnic exposure was conducted under hyperoxic conditions before and after $\mathrm{CNO}$ administration. Breathing parameters were measured during sequential treatments with $100 \% \mathrm{O}_{2}, 5 \% \mathrm{CO}_{2}, 8 \% \mathrm{CO}_{2}$, CNO $(100 \%$ $\mathrm{O}_{2}$ ), CNO plus $5 \% \mathrm{CO}_{2}$ and plus $8 \% \mathrm{CO}_{2}$ in each mouse. Ventilatory flow signals were recorded, amplified, digitized, and analyzed using IOX 2.7 (EMKA Technologies) to determine ventilatory parameters over sequential $20 \mathrm{~s}$ epochs ( $\sim 50$ breaths). The response to hypercapnia was determined from the peak MV (20 s epoch) following the hypercapnic exposure. At the end of the experiments, some animals were anesthetized with an overdose of pentobarbital and perfused transcardially for histological experiments.

Ablation of Phox $2 b$-expressing neurons. Microinjections of the viral vector into the NTS were performed using the similar protocol as depicted above. Shortly, Cre-dependent AAV vectors encoding a genetically engineered Casp3 gene (AAV-CAG-DIO-taCasp3-TEVp, $100 \mathrm{nl}$ per injection, 4 injections) and mCherry (AAV-CAG-DIO-mCherry, control vector) were bilaterally injected into the intermediate and caudal NTS, respectively. Mice were then given a 4 week recovery before plethysmography experiments. To validate ablation of Phox $2 \mathrm{~b}$-expressing neurons, the quantitative PCR ( $\mathrm{PPCR}$ ) was performed to analyze the level of Phox $2 b$ mRNA in the NTS. Shortly, after anesthetization, the NTS tissues of mice were rapidly dissected out and preserved in lysate solution. RNA purification columns (Eastep Super total RNA extract kit, Promega) were used to purify RNA, followed by reverse transcription (Super Script III First-Strand Synthesis System for RT-PCR, Invitrogen). The qPCR was performed on ABI Quant Studio 6 flex system $\left(1\right.$ cycle at $50^{\circ} \mathrm{C}$ for $2 \mathrm{~min}$; 1 cycle at $95^{\circ} \mathrm{C}$ for $10 \mathrm{~min} ; 40$ cycles at $95^{\circ} \mathrm{C}$ for $15 \mathrm{~s}$ and $60^{\circ} \mathrm{C}$ for $1 \mathrm{~min}$; 1 cycle at $95^{\circ} \mathrm{C}$ for $15 \mathrm{~s} ; 1$ cycle at $60^{\circ} \mathrm{C}$ for $1 \mathrm{~min} ; 1$ cycle at $95^{\circ} \mathrm{C}$ for $15 \mathrm{~s}$ ) using SYBR Green reaction mix (Applied Biosystems). GAPDH was used as an internal reference. The following primers were used: GAPDH forward primer: 5'-GCAAATTCAACGGCACAGTCAAGG-3', reverse primer: 5'-TCTCGTGGTTCACACCCATCACAA-3'; Phox $2 \mathrm{~b}$ forward primer: 5'-TACGCCGCAGTTCCATACAAACTC-3', reverse primer: 5'-TCTTTGAGCTGCG CGCTTGTGAAG-3'.

Histology and immunoassaying. The immunohistochemical protocol has been depicted previously (Kumar et al., 2015). Briefly, mice were deeply anesthetized with urethane $(1.8 \mathrm{~g} / \mathrm{kg}$, i.p. $)$ and transcardially perfused with $200 \mathrm{ml}$ of chilled saline, followed by $4 \%$ phosphate-buffered PFA (0.1 M, pH 7.4). The mouse was decapitated, and the brainstem was dissected out, stored in the perfusion fixative at $4^{\circ} \mathrm{C}$ for $48 \mathrm{~h}$, and immersed in $30 \%$ sucrose in PBS at $4^{\circ} \mathrm{C}$ for at least $2 \mathrm{~d}$. A series of $25 \mu \mathrm{m}$ sections was obtained with a cryostat (CM1950; Leica Microsystems). The tissue sections were washed with PBS for 3 times, and then immersed in blocking buffer ( $2 \% \mathrm{BSA}$ in PBS) for $30 \mathrm{~min}$ at room temperature. For immunofluorescence staining, the sections were incubated with primary antibodies at $4^{\circ} \mathrm{C}$ overnight. After rinsing with PBS again, the sections were then incubated with secondary antibodies for $1 \mathrm{~h}$ at room temperature. Finally, the sections were mounted and examined with a fluorescence microscope (DM6000B, Leica Microsystems) or confocal microscope (LSM800, Carl Zeiss).

For cFos-based histological analysis of CNO-stimulated NTS neurons, the hM3Dq-injected mice were placed in the chamber, exposed to $100 \%$ $\mathrm{O}_{2}$, and then intraperitoneally injected with $\mathrm{CNO}(1 \mathrm{mg} / \mathrm{kg})$. After 45 
A

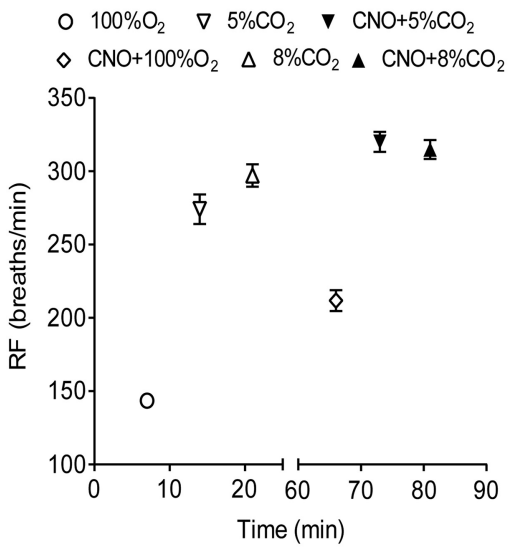

C

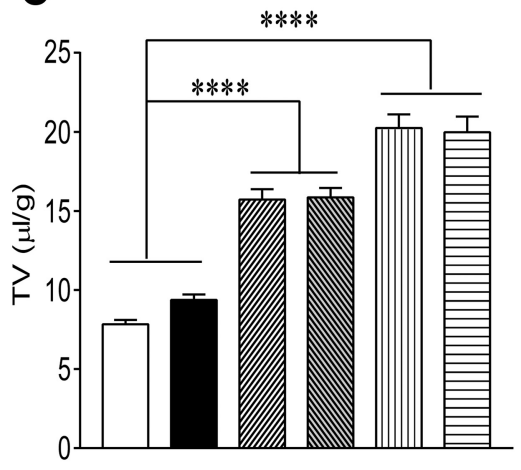

B

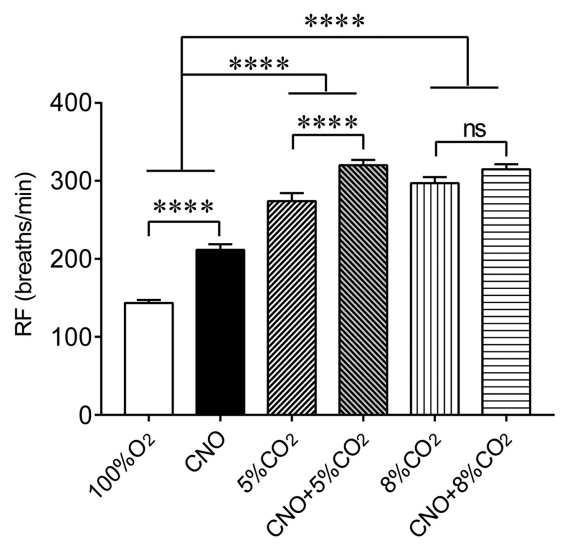

D

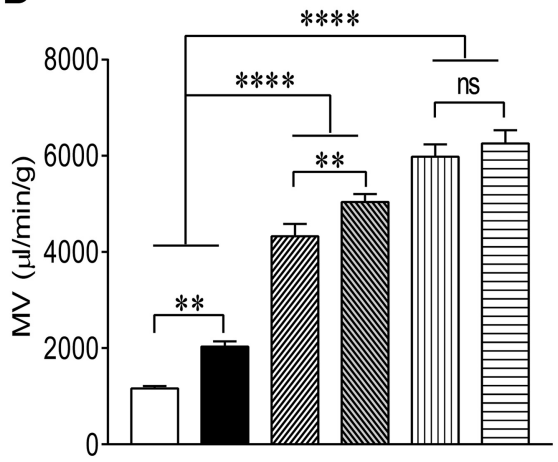

Figure 4. Additive effect of $\mathrm{CNO}$ and $\mathrm{CO}_{2}$ stimulations on pulmonary ventilation. $A$, Schematic diagram depicting the experimental protocol. RF was measured in response to sequential treatments with $100 \% \mathrm{O}_{2}, 5 \%$ and $8 \% \mathrm{CO}_{2}, \mathrm{CNO}, \mathrm{CNO}$ plus $5 \%$ and plus $8 \% \mathrm{CO}_{2}$, respectively. The protocol was also applied to TV and MV. $\boldsymbol{B}-\boldsymbol{D}$, Effects of sequential stimulations on breathing parameters. Notably, the combined application of $5 \% \mathrm{CO}_{2}$ and $\mathrm{CNO}$ elicited greater increases in $\mathrm{RF}$ and $\mathrm{MV}$ than $5 \% \mathrm{CO}_{2}$ alone, with unchanged TV, whereas insignificant difference in breathing parameters was found between $8 \% \mathrm{CO}_{2}$ alone and $8 \% \mathrm{CO}_{2}$ plus $\mathrm{CNO}$. Numbers of mice: $n=18$ (except 9 of 18 mice for $8 \%\left(\mathrm{C}_{2}\right)$. One-way ANOVA with Holm-Sidak's multiple-comparisons test: ${ }^{* *} p<0.01$; **** $p<0.0001$. ns, no significance.

min, the mice were anesthetized and perfused transcardially with fixative for subsequent histological processing as described above. For cFosbased histological analysis of the proportion of $\mathrm{CO}_{2}$-activated mCherrytransduced NTS neurons, mice were placed in the chamber and correspondingly exposed to $100 \% \mathrm{O}_{2}$ or $5 \% \mathrm{CO}_{2}$ or $8 \% \mathrm{CO}_{2}$ (balance $\mathrm{O}_{2}$ ) for $50 \mathrm{~min}$, followed by $100 \% \mathrm{O}_{2}$ for $60 \mathrm{~min}$. The mice were then killed for histological experiments. For cFos-based histological analysis of whether chemogenetic stimulation of Phox $2 b$-expressing NTS neurons affected neuronal activity of preBötzinger complex (preBötC), the transcardial perfusion and histological protocols were performed 120 min after injections of $\mathrm{CNO}$ or saline in hM3Dq-injected mice.

To calculate the proportion of $\mathrm{CO}_{2}$ - or CNO-stimulated mCherrytransduced neurons in the NTS, cell counts were performed in 6 coronary sections of each mouse. The rostrocaudal position of each coronary section was determined (bregma: -7.20 to $-7.76 \mathrm{~mm}$, approximately corresponding to intermediate and caudal NTS) according to the atlas of The mouse brain in stereotaxic coordinates (Paxinos and Franklin, 2003). To count cFos-expressing preBötC neurons, five consecutive coronary sections (bregma: -6.72 to $-7.20 \mathrm{~mm}$, separation by $90 \mu \mathrm{m}$ ) were collected for histology. The Phox $2 \mathrm{~b}$ staining was conducted together with cFos to easily determine the location of preBötC neurons.

The cFos was detected with a rabbit antibody (dilution 1:400, Cell Signaling Technology, catalog \#2250, RRID:AB_2247211) and corresponding secondary antibody (dilution 1:200, Jackson ImmunoResearch Laboratories, catalog \#711-095-152, RRID:AB_2315776). The Phox2b was detected with a mouse monoclonal antibody (dilution 1:200, sc376993; Santa Cruz Biotechnology) that was validated previously (Fu et al., 2017) and revealed with goat anti-mouse FITC (dilution 1:200,
Jackson ImmunoResearch Laboratories, cata$\log$ \#115-095-146, RRID:AB_2338599) or goat anti-mouse Cy3 (dilution 1:200, Jackson ImmunoResearch Laboratories catalog \#115165-003, RRID:AB_2338680). Images were acquired and processed with the Application Suit (Leica Microsystems).

Slice preparation and electrophysiological recordings. Protocols have been described previously in detail (Wang et al., 2013b). Briefly, the mice that received AAV-EF1 $\alpha$-DIO-hM3DqmCherry were deeply anesthetized with $5 \%$ pentobarbital sodium $(1.5 \mathrm{ml} / \mathrm{kg})$ and decapitated. Brainstem coronary slices (220-250 $\mu \mathrm{m}$, thickness) were cut quickly, recovered for $1 \mathrm{~h}$, and subsequently transferred into a recording chamber for electrophysiological experiments. The mCherry-labeled neurons were chosen and whole-cell recordings were made using a Multiclamp 700B amplifier and a Digidata $1440 \mathrm{~A}$ analog-to-digital converter (Molecular Devices). CNO was bath-applied at a final concentration of $30 \mu \mathrm{M}$. Recordings of membrane potential were obtained by wholecell current clamp.

Statistics. Statistical analysis was done using Prism (GraphPad Prism, RRID:SCR_002798). Values were reported as mean \pm SEM. To compare the differences within and between groups, we used paired or unpaired Student's $t$ test, or one- or two-way ANOVA with the appropriate post hoc tests. Differences with $p<$ 0.05 were considered statistically significant.

\section{Results}

Validation of the chemogenetic approach

We used a previously generated BAC transgenic mouse line expressing Cre recombinase under the control of Phox $2 b$ specific promoter (Scott et al., 2011). To chemogenetically activate Phox $2 \mathrm{~b}-$ expressing NTS neurons in vivo, Cre-inducible AAV vectors encoding hM3Dq were microinjected into the NTS in Phox2b-Cre mice (Fig. 1A). The phenotype of the hM3Dq-mCherrytransduced neurons was examined by immunohistochemistry. As shown in Figure $1 B, C$, based on cell counts obtained in 6 mice, the mCherry ${ }^{+}$Phox $2 \mathrm{~b}^{+}$neurons accounted for $\sim 83 \%$ of total number of mCherry ${ }^{+}$neurons and for $\sim 13 \%$ of Phox $2 b^{+}$neurons. In addition, the majority of transduced neurons $(\sim 94 \%)$ were located in the NTS (Fig. $1 D-F)$, so the remaining $(\sim 6 \%)$ in dorsal motor nucleus of vagus (data not shown).

To validate the transduction and functionality of the hM3Dq vector, histological and electrophysiological assays were performed. Four weeks after injection of AAV-EF1 $\alpha$-DIO-hM3DqmCherry, mice were transcardially perfusion-fixed $45 \mathrm{~min}$ after treatment with the hM3Dq activator CNO $(1 \mathrm{mg} / \mathrm{kg}$, i.p.) or equal volume of saline. The hM3Dq activation of Phox $2 b-$ expressing NTS neurons was confirmed by showing immunoreactivity for $\mathrm{cFos}\left(\mathrm{cFos}^{+}\right)$following $\mathrm{CNO}$ administration. Apparently, the number of CNO-stimulated $\mathrm{cFos}^{+} \mathrm{mCherry}^{+}$ neurons was far greater compared with that treated with saline $\left(n=5\right.$ for each, $t_{(8)}=9.371, p<0.0001$; Fig. $\left.2 A-G\right)$. In electrophysiological experiments, acute brainstem slices were prepared and whole-cell patch-clamp recordings were made to test changes in membrane potential in hM3Dq-mCherry transduced neurons. Bath application of $\mathrm{CNO}(30 \mu \mathrm{M})$, instead 
of saline, produced a rapid reversible depolarization $\left(n=9,6.0 \pm 1.0 \mathrm{mV}, t_{(8)}=\right.$ 6.07, $p=0.0003$; Fig. $2 H, I)$, indicating the excitatory effect of CNO on the transduced neurons. Collectively, these data confirm the high efficacy of chemogenetic activation of Phox $2 \mathrm{~b}$-expressing neurons.

\section{Chemogenetic stimulation of Phox $2 b-$} expressing neurons activates breathing We next applied this chemogenetic approach to Phox2b-Cre mice transduced with hM3Dq or a control vector (AAVEF1 $\alpha$-DIO-mCherry). Four weeks after vector injection, we performed plethysmography in conscious mice when they were quietly resting. Breathing parameters, including RF, TV, MV, $\mathrm{T}_{\mathrm{i}}$, and $\mathrm{T}_{\mathrm{e}}$, were compared between $\mathrm{CNO}$ and saline injections in hM3Dq-transduced Phox 2 b-Cre mice ( $n=22$ for saline, $n=$ 32 for $\mathrm{CNO}$ ). As shown in Figure $3 A$, injection of $\mathrm{CNO}$, rather than saline, produced a long-lasting increase in RF. Such an increase commenced $15 \mathrm{~min}$ after the injection, peaked during 30-60 $\mathrm{min}$ (143 \pm 5 vs $192 \pm 5$ breaths/min, saline vs $\mathrm{CNO}$ at $\sim 45 \mathrm{~min}, F_{(1,405)}=59.97, p<$ 0.0001 ), then returned to baseline level at $\sim 180 \mathrm{~min}$. This remarkably stimulatory effect of CNO on RF persisted for at least 90 min. In contrast, no significant change in TV was found between two groups (Fig. $3 B)$. Thereby, the increase in MV $(1479 \pm 63$ vs $2219 \pm 78 \mu \mathrm{l} /$ $\mathrm{min} / \mathrm{g}$, saline vs $\mathrm{CNO}$ at $\sim 45 \mathrm{~min}, F_{(1,405)}=61.83, p<0.0001$; Fig. $3 C$ ) was solely due to an increase in RF. Meanwhile, administration of $\mathrm{CNO}$, instead of saline, caused significant decreases in $\mathrm{T}_{\mathrm{i}}\left(99 \pm 4\right.$ vs $84 \pm 2 \mathrm{~ms}$, saline vs $\mathrm{CNO}$ at $\sim 45 \mathrm{~min}, F_{(1,102)}=$ $6.193, p=0.0006$; Fig. $3 D)$ and $\mathrm{T}_{\mathrm{e}}(377 \pm 13 \mathrm{vs} 253 \pm 9 \mathrm{~ms}$, saline vs $\mathrm{CNO}$ at $\sim 45 \mathrm{~min}, F_{(1,102)}=9.953, p<0.0001$; Fig. $\left.3 E\right)$. In control vector-injected mice (mCherry expression only), neither $\mathrm{CNO}$ nor saline produced significant changes in breathing parameters (data not shown). Thus, we conclude that selective stimulation of Phox 2 b-expressing NTS neurons potentiates baseline pulmonary ventilation.

\section{Additive effects of $\mathrm{CNO}$ and $\mathrm{CO}_{2}$ stimulations}

Having assessed respiratory effects of Phox $2 \mathrm{~b}$-expressing neurons, we further sought to determine whether chemogenetic activation of these neurons synergized with $\mathrm{CO}_{2}$ stimulation to strengthen a ventilatory response. As illustrated in Figure $4 A$, breathing parameters were measured during sequential treatments with $100 \% \mathrm{O}_{2}, 5 \% \mathrm{CO}_{2}, 8 \% \mathrm{CO}_{2}$, $\mathrm{CNO}\left(100 \% \mathrm{O}_{2}\right), \mathrm{CNO}$ plus $5 \% \mathrm{CO}_{2}$ and plus $8 \% \mathrm{CO}_{2}$ in each mouse $(n=18$, except 9 of 18 mice for $8 \% \mathrm{CO}_{2}$ ). Each measurement was made near the end of each experimental epoch. The time point for $\mathrm{CNO}$ effect was chosen based on the above results indicating that the strongest ventilatory response to $\mathrm{CNO}$ occurred at $\sim 45 \mathrm{~min}$. As shown in Figure 4, compared with exposure to $100 \% \mathrm{O}_{2}$, CNO injection, exposure to $5 \%$ or $8 \% \mathrm{CO}_{2}$ (balance $\mathrm{O}_{2}$ ) all produced an obvious increase in $\operatorname{RF}\left(F_{(5,84)}=88.41, p<0.0001\right.$ for both $) ; 5 \%$ and $8 \%$ $\mathrm{CO}_{2}$ rather than $\mathrm{CNO}$ also notably increased the $\mathrm{TV}\left(F_{(5,84)}=\right.$ $64.8, p<0.0001$ for $5 \%$ and $8 \% \mathrm{CO}_{2}, p=0.1935$ for CNO; Fig.
B

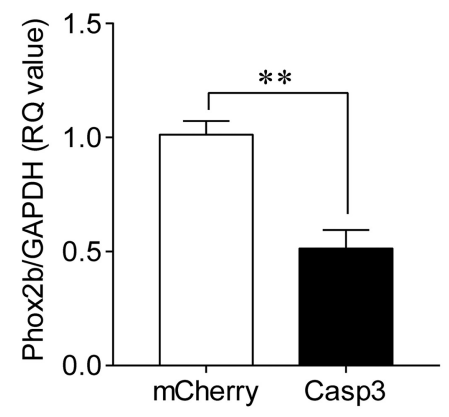

E

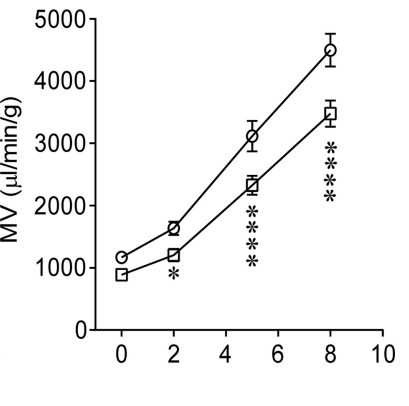

Figure 5. Effect of ablation of Phox2b-expressing neurons on hypercapnic ventilatory responses. $A$, Schematic diagrams illustrating a delivery of Cre-dependent Casp3 into the NTS. $\boldsymbol{B}$, Quantitative analysis of Phox2b mRNA to validate selective ablation of hox 2 b-expressing neurons using qPCR. $n=9$ samples from 3 mice for mCherry; $n=12$ samples from 4 mice for Casp3. ${ }^{* *} p<$ ( Ext multiple-comparisons test: ${ }^{*} p<0.05 ;{ }^{* *} p<0.01 ;{ }^{* * *} p<0.001 ;{ }^{* * * *} p<0.0001$.

$4 C)$. Consequently, all the above stimulations considerably increased the MV alone $\left(F_{(5,84)}=119.2, p<0.0001\right.$ for all except $p=0.002$ for $\mathrm{CNO}$; Fig. $4 D)$. The combined application of $\mathrm{CNO}$ and $5 \% \mathrm{CO}_{2}$ produced a greater increase in $\mathrm{RF}(p<0.0001)$ and $\mathrm{MV}$ $(p=0.0081)$ than $5 \% \mathrm{CO}_{2}$ only, with insignificant difference in TV $\left(p=0.9662\right.$; Fig. $4 C$ ). However, exposure to $8 \% \mathrm{CO}_{2}$ plus $\mathrm{CNO}$ caused similar increases in RF, TV, and MV compared with $8 \% \mathrm{CO}_{2}$ alone ( $p>0.05$ for all). Overall, chemogenetic stimulation of Phox 2b-expressing NTS neurons synergizes with a moderate level of $\mathrm{CO}_{2}$ stimulation to enhance the ventilatory response.

\section{Effect of ablation of Phox2b-expressing neurons on hypercapnic ventilation}

Our prior study has demonstrated that microinjection of a neurotoxin in the NTS to moderately destroy Phox2b-expressing neurons impaired a hypercapnic ventilatory response in conscious mice (Fu et al., 2017). To further verify whether this set of neurons was required for the central respiratory chemoreflex, we performed a loss-of-function study that used a genetic approach to ablate these neurons in Phox2b-Cre transgenic mice. To that end, the AAV-CAG-DIO-taCasp3-TEVp (AAV-CAG-DIOmCherry for control) was injected in the NTS to selectively ablate Phox $2 \mathrm{~b}$-expressing neurons ( $n=10$ for each group; Fig. $5 A$ ). The Casp3 activation has been evidenced to induce cell apoptosis (Resch et al., 2017; Zhao et al., 2017). As shown in Figure 5B, the Phox $2 \mathrm{~b}$ mRNA level in the NTS was significantly reduced $(\sim 50 \%)$ in Casp3-injected mice $(n=4)$ than mCherry-injected mice $(n=3, p=0.0018$, mCherry vs Casp3; Fig. $5 B)$, in favor of a moderate ablation of Phox $2 \mathrm{~b}$-expressing neurons. During exposure to $100 \% \mathrm{O}_{2}$, loss of Phox $2 \mathrm{~b}$-expressing neurons did not 
A

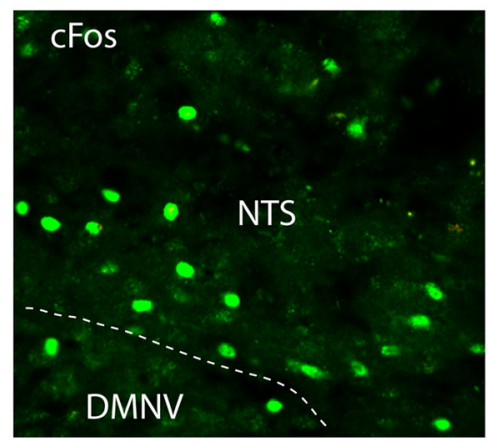

D

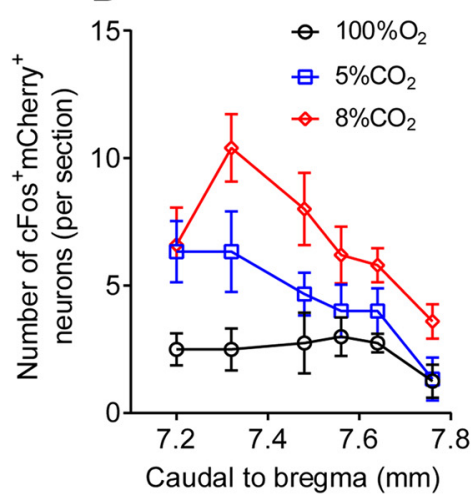

B

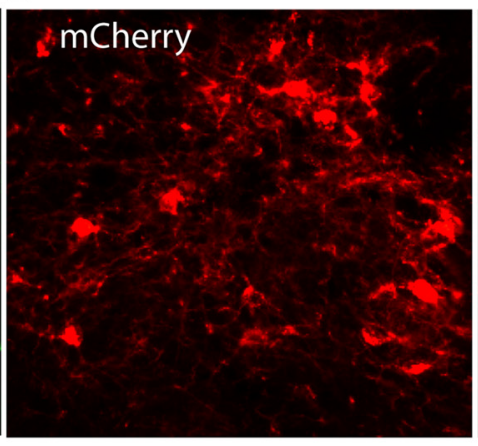

$E$

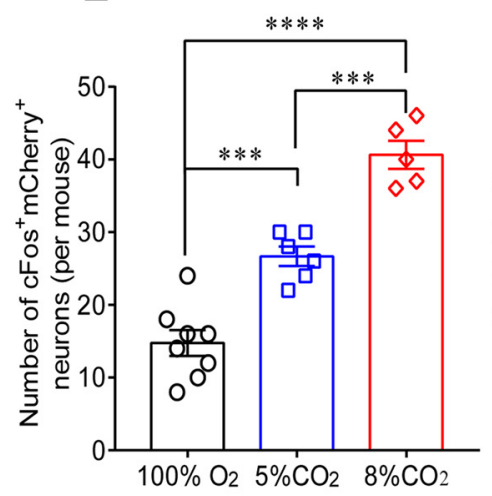

C

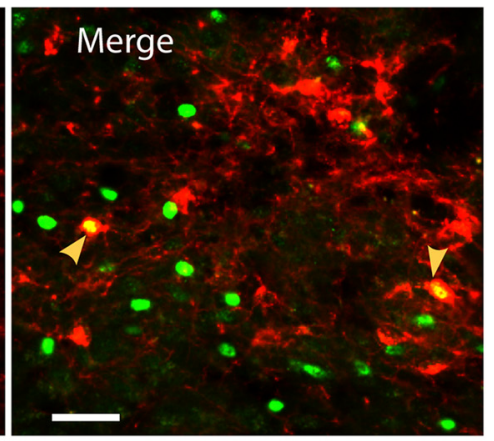

$\mathbf{F}$

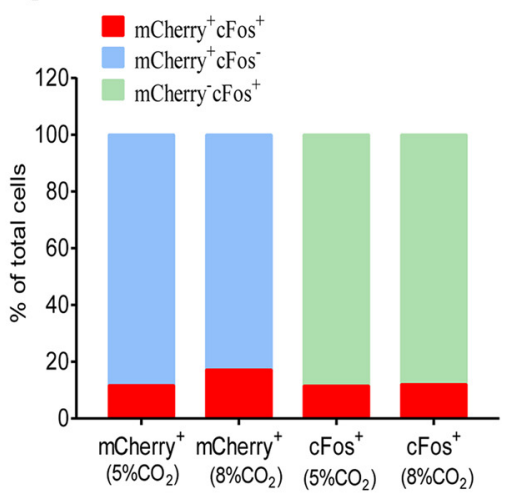

Figure 6. $\mathrm{CO}_{2}$ sensitivity of Phox2b-expressing neurons. $\boldsymbol{A}-\boldsymbol{C}$, Immunofluorescence images showing expression of $\mathrm{CFos}_{\text {and }} \mathrm{mCherry.} \mathrm{The} \mathrm{mouse} \mathrm{was} \mathrm{exposed} \mathrm{to} 8 \%$ C $\mathrm{O}_{2}$ for 50 min before immunohistological experiments. $\mathrm{CO}_{2}$-activated neurons were denoted by the immunoreactivity for $\mathrm{CFos}\left(\mathrm{green}, \boldsymbol{A}\right.$ ). The $\mathrm{CFos}^{+} \mathrm{mCherry}^{+}$neurons (denoted by arrows, $\boldsymbol{C}$ ) represented $\mathrm{CO}_{2}{ }^{-}$ activated transduced neurons. Scale bar, $25 \mu \mathrm{m}$. D, Rostrocaudal distribution of $\mathrm{CFos}^{+} \mathrm{mCherry}^{+}$neurons in 6 coronary sections from each mouse exposed to different concentrations of $\mathrm{CO}_{2}$. Number of mice: $n=8$ for $100 \% 0_{2} ; n=6$ for $5 \% \mathrm{CO}_{2} ; n=5$ for $8 \% \mathrm{CO}_{2} . \boldsymbol{E}$, Comparisons in the average number of cFos ${ }^{+} \mathrm{mCherry}{ }^{+}$neurons of each group based on cell counts in $\boldsymbol{D}$. $\boldsymbol{F}$, Proportion of $\mathrm{CFos}^{+} \mathrm{mCherry}^{+}$neurons in the total number of mCherry ${ }^{+}$or $\mathrm{CFos}^{+}$neurons. The calculations were made based on cell counts in $\boldsymbol{E}$. ${ }^{* * *} p<0.001$ (one-way AN0VA with Tukey's test). ${ }^{* * * *} p<$ 0.0001 (one-way ANOVA with Tukey's test).

affect baseline TV and MV, with the exception that RF was lower in Casp3-injected mice compared with mCherry-injected mice $\left(F_{(1,9)}=4.933, p=0.0061\right.$; Fig. $\left.5 C\right)$. Exposure to $2 \% \mathrm{CO}_{2}$ remarkably increased the RF and MV in both groups, but the responses of RF and MV were reduced in Casp3-injected mice compared with the counterpart $\left(p=0.0127\right.$ for RF; $F_{(1,9)}=$ $11.11, p=0.0353$ for MV). When challenged by $5 \%$ and $8 \% \mathrm{CO}_{2}$, although both TV and MV were apparently increased in two groups, the changes in two parameter values were markedly reduced in Casp3-injected mice relative to mCherry-injected mice $\left(\mathrm{TV}, F_{(1,9)}=3.913, p=0.0007\right.$ for $5 \% \mathrm{CO}_{2}, p<0.0001$ for $8 \%$ $\mathrm{CO}_{2}$; MV, $p<0.0001$ for both; Fig. $\left.5 D, E\right)$, with insignificant difference in RF (Fig. 5C). Together, we confirm that Phox2bexpressing NTS neurons are required for the hypercapnic ventilatory response.

\section{$\mathrm{CO}_{2}$ sensitivity of Phox $2 \mathrm{~b}$-expressing neurons}

The Phox $2 \mathrm{~b}$-expressing neurons stimulated by the chemogenetic approach are probably $\mathrm{CO}_{2}$-activated as implicated previously and act as central respiratory chemoreceptors (Fu et al., 2017). To determine whether hM3Dq-transduced neurons are $\mathrm{CO}_{2}$ sensitive, the immunoreactivity to $\mathrm{cFos}$ was examined in mCherry-transduced mice subjected to inhaling $100 \% \mathrm{O}_{2}(n=$ $8), 5 \%(n=6)$ and $8 \% \mathrm{CO}_{2}(n=5)$ for $50 \mathrm{~min}$, respectively. As shown in Figure $6 A-C$, photographs indicate cFos (green) and mCherry (red) and coexpression (denoted by yellow arrows). Cell counts were conducted in 6 coronary sections from each mouse (Fig. $6 D$ ). Figure $6 E$ demonstrates that the total number of
$\mathrm{cFos}^{+} \mathrm{mCherry}^{+}$neurons in mice treated with $8 \% \mathrm{CO}_{2}(41 \pm 2)$ is greater than that with $5 \% \mathrm{CO}_{2}\left(21 \pm 1, F_{(2,16)}=54.52, p=\right.$ $0.0002)$ and $100 \% \mathrm{O}_{2}(15 \pm 2, p<0.0001)$, so is the significant difference between $5 \% \mathrm{CO}_{2}$ and $100 \% \mathrm{O}_{2}(p=0.0003)$, suggesting the presence of a fraction of $\mathrm{CO}_{2}$-activated Phox $2 \mathrm{~b}$ expressing neurons. After data normalization, 5\% and $8 \%$ $\mathrm{CO}_{2}$-activated $\mathrm{cFos}^{+} \mathrm{mCherry}^{+}$neurons correspondingly account for $\sim 12 \%$ and $18 \%$ of all the mCherry $^{+}$neurons, as well as for $\sim 12 \%$ and $12 \%$ of all the $\mathrm{cFos}^{+}$neurons (Fig. $6 \mathrm{~F}$ ). Hence, a small subset of mCherry-transduced neurons is $\mathrm{CO}_{2}$-activated and presumably functions as central respiratory chemoreceptors.

\section{Selective stimulation of Phox $2 \mathrm{~b}$-expressing neurons activates preBötC neurons}

To examine whether chemogenetic stimulation of Phox2bexpressing NTS neurons increased baseline ventilation through activation of $\mathrm{rCPG}$, the neuronal activity of preBötC was assessed by counting cFos-expressing cells. Cell counts were made in 5 consecutive coronary sections in hM3Dq-transduced mice treated with either saline or CNO ( $n=4$ for each group). The number of $\mathrm{CFos}^{+}$neurons is larger in the $\mathrm{CNO}$ group than in the saline group $\left(52 \pm 6\right.$ vs $105 \pm 5$, saline vs $\mathrm{CNO}, t_{(6)}=8.803, p=$ 0.0001 ; Fig. $7 A$ ). As shown in typical confocal images in Figure $7 B-D, \mathrm{cFos}^{+}$(green) neurons in the preBötC are located in ventral side of nucleus ambiguus. In addition, Phox $2 \mathrm{~b}$ (red) is not expressed in $\mathrm{cFos}^{+}$neurons of preBötC. Apparently, the number of preBötC neurons activated by $\mathrm{CNO}$ is greater compared with saline injection. Thus, the present result suggests a circuit mech- 
A
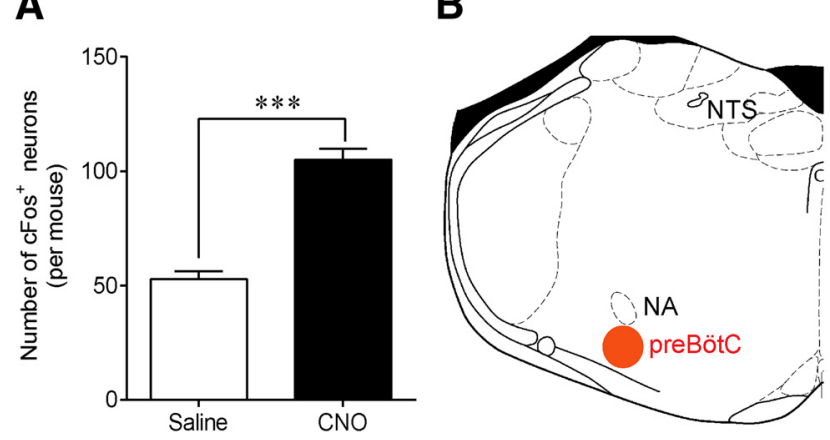

C

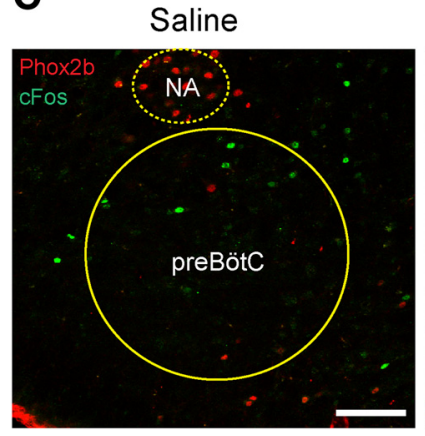

D

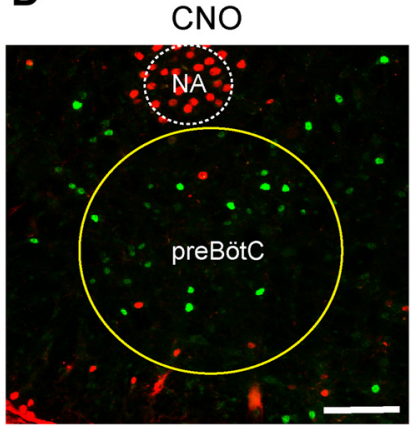

Figure 7. Stimulation of Phox2b-expressing neurons activates preBötC neurons. $A$, Effect of Phox2b-expressing NTS neuron activation on neuronal activity of preBötC. The activated preBötC neurons were indicated by immunoreactivity to cFos. Cell counts were made in 5 coronary sections ( $25 \mu \mathrm{m}$ thick; $6.72-7.20 \mathrm{~mm}$ caudal to bregma) in each hM3Dq-injected mouse treated with either CNO or saline. $n=4$ for each group. ${ }^{* *} p<0.001$ (unpaired $t$ test). $\boldsymbol{B}$, Schematic diagram indicating the coronary plane of a preBötC-containing medullary section ( $\sim 7 \mathrm{~mm}$ caudal to bregma) corresponding to $\boldsymbol{C}$ and $\boldsymbol{D}$. C, $\boldsymbol{D}$, Confocal images showing CFosexpressing preBötC neurons in mice treated with saline or CNO. Phox2b staining (red) was performed together with $\mathrm{CFos}$ (green) to assist with pinpointing the preBötC. No overlap of cFos and Phox2b was observed in preBötC cells. NA, Nucleus ambiguus. Scale bar, $100 \mu \mathrm{m}$.

anism by which selective stimulation of Phox $2 b$-expressing NTS neurons modulates the activity of preBötC neurons and finally potentiates baseline ventilation.

\section{Projection of Phox $2 b$-expressing neurons to ventrolateral medulla}

We further confirmed the neuroanatomical connections between Phox2b-expressing NTS neurons and ventral medullary areas involved in respiratory rhythm generation. As illustrated in Figure $8 D-F$, mCherry expression was evident, not only in neuronal somata, but also in the axons traveling from dorsal medulla to ventrolateral sides. Numerous axons from Phox $2 \mathrm{~b}$-expressing neurons terminated in respiratory compartments at three levels of coronary planes, approximately corresponding to BötC (Fig. $8 A, D$ ), preBötC (Fig. $8 B, E$ ), and the rostral part of ventral respiratory group (rVRG; Fig. $8 C, F$ ). Despite no functional evidence supporting the direct synaptic transmissions between mCherrytransduced axons and postsynaptic neurons from rCPG, we would confirm that these axons were directly projected from transduced NTS neurons because the viral vector used for tracing does not act transsynaptically. This projection pattern indicates that activation of Phox $2 \mathrm{~b}$-expressing NTS neurons could modulate the activity of rCPG via direct synaptic connections.

\section{Discussion}

We demonstrate that chemogenetic activation of Phox2bexpressing NTS neurons increases baseline MV via an increase in
$\mathrm{RF}$ in conscious mice. In addition, Phox $2 \mathrm{~b}$-expressing neuron activation synergizes with moderate level of $\mathrm{CO}_{2}$ stimulation to enhance pulmonary ventilation. In contrast, selective ablation of Phox 2 b-expressing neurons attenuates the hypercapnic ventilatory response. Moreover, chemogenetic stimulation of this set of neurons enhances neuronal activity in the preBötC. Finally, we present the neuroanatomical evidence of the direct projection of Phox2b-expressing NTS neurons to rCPG.

\section{Selective stimulation of Phox2b-expressing neurons potentiates baseline ventilation}

Here, we attribute the chemogenetic effect on breathing to Phox $2 \mathrm{~b}$-expressing neuron activation based on the following reasons. First, the majority of mCherry-transduced neurons ( $>83 \%$ ) were Phox 2 b-positive, validating the high selectivity of viral transfection. Second, the mCherry-transduced neurons were primarily located in the NTS with a very small proportion in the dorsal motor nucleus of vagus. Third, immunohistological and electrophysiological experiments showed that CNO effectively activated mCherry-transduced neurons.

In the present study, chemogenetic activation of Phox $2 b-$ expressing NTS neurons remarkably increased baseline MV solely due to an increase in RF. Similar studies in the NTS have been performed previously. An early report (Nattie and Li, 2002) found that $\mathrm{CO}_{2}$ dialysis in the NTS increased both RF and TV in awake rats; however, the neurochemical phenotypes of these NTS cells were unknown. The present study implicated the Phox $2 \mathrm{~b}$ expressing neurons, which are definitely associated with CCHS. Recently, Huda et al. (2012) found that blockade of acid-sensitive ion channels in the NTS strongly reduced $\mathrm{CO}_{2}$-stimulated increase in burst frequency of the phrenic bursting activity in anesthetized rats. Consistent with our prior study demonstrating that chronic lesions of Phox $2 \mathrm{~b}$-containing NTS neurons did not affect resting MV but strongly reduced $\mathrm{CO}_{2}$-stimulated increases in TV and MV (Fu et al., 2017), the present finding also showed that selective ablation of Phox2b-expressing neurons impaired the hypercapnic ventilatory response primarily via affecting TV. The above effects can be summarized in three modes: the NTS regulates (1) RF only, (2) respiratory amplitude only, and (3) both RF and amplitude.

Here we emphasize the consequence of respiratory output in response to Phox $2 \mathrm{~b}$-expressing neuron stimulation, although such effects may be achieved through altering either RF or TV, or both. So far, it seems difficult to perform a molecular dissection of RF- and amplitude-regulating neurons. The two parameters may be differentially modulated by the following factors: neurochemical phenotype of neurons involved, anesthetized versus conscious animals, normocapnic versus hypercapnic conditions, and gain-of-function versus loss-of-function experiments. In the present study, the Phox $2 \mathrm{~b}$ neuron-stimulated potentiation of MV via mobilizing RF suggests a resting RF reserve at the level of NTS. In animals with intact neural circuits in either NTS or RTN, exposure to $\mathrm{CO}_{2}$ increased MV via mobilizing both RF and TV (Nattie and Li, 2002; Kumar et al., 2015; Fu et al., 2017). The $\mathrm{CO}_{2}$-stimulated ventilatory response may involve activation of several types of neurons, such as glutamatergic, GABAergic, and catecholaminergic neurons. In loss-of-function experiments, the stimulatory effect of $\mathrm{CO}_{2}$ on RF or TV may be diverse because of perturbation of neural circuits within the NTS or between the NTS and rCPG. Our prior and present findings show that ablation of Phox $2 b$ neurons attenuated the hypercapnic ventilatory response primarily via a reduction of TV rather than RF. Similar results were observed in the locus ceruleus. Selective lesions of LC 

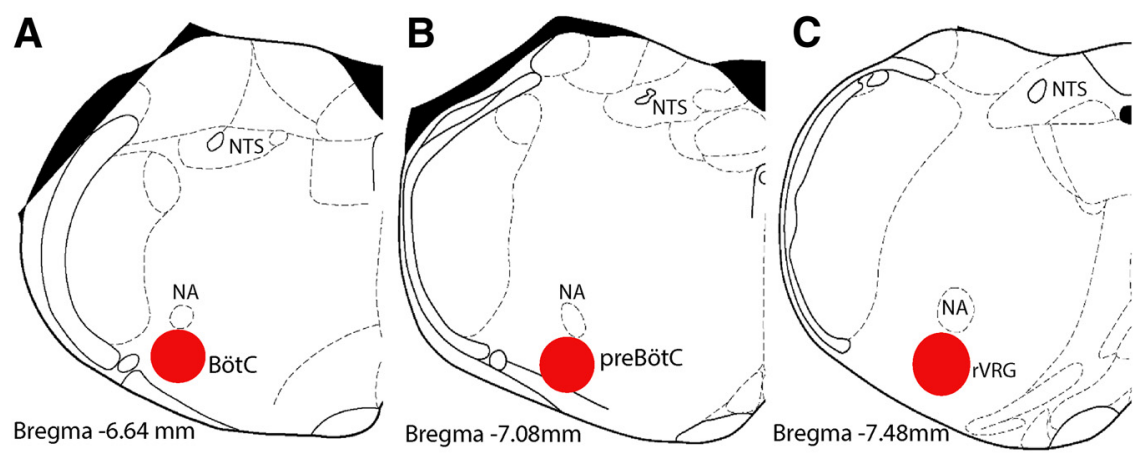

D

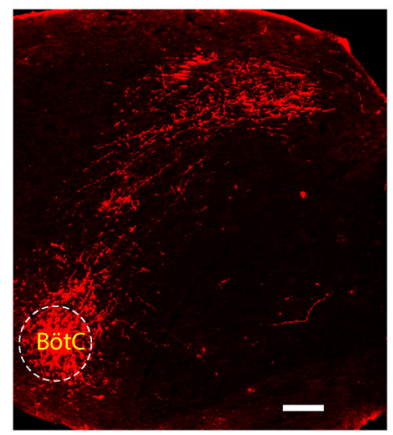

E

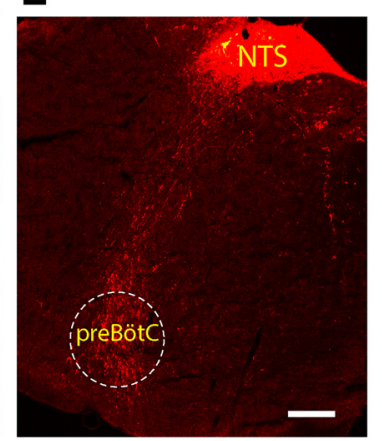

$F$

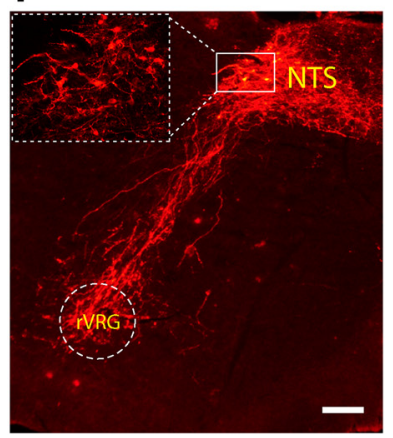

Figure 8. Projection of Phox2b-expressing neurons to the rCPG. $\boldsymbol{A}-\boldsymbol{C}$, Schematic profile illustrating the location of BötC $(\boldsymbol{A})$, preBötC $(\boldsymbol{B})$, and rVRG $(\boldsymbol{C})$ in brainstem coronary sections. $\boldsymbol{D}-\boldsymbol{F}$, Photomicrographs showing the projection of mCherry-transduced neurons to the ventrolateral medulla, approximately corresponding to BötC $(\boldsymbol{D})$, preBötC $(\boldsymbol{E})$, and rVRG $(\boldsymbol{F})$. The details in lined rectangle were enlarged in a dashed rectangle in $\boldsymbol{F}$. Scale bar, $200 \mu \mathrm{m}$.

noradrenergic neurons significantly blunted hypercapnic ventilatory responses via a reduced TV instead of RF (Biancardi et al., 2008); however, in another study, severe destruction of locus ceruleus catecholaminergic neurons produced an inhibitory effect on the hypercapnic ventilatory response via a diminished RF rather than TV (Li and Nattie, 2006). Thus, more studies are required for molecular dissection of neural circuits involved in differential regulation of pulmonary ventilation.

\section{Phox2b-expressing neurons are required for central respiratory chemoreflex}

The loss-of-function experiment demonstrates that selective ablation of Phox $2 \mathrm{~b}$-expressing neurons attenuated the hypercapnic ventilatory response. Chemosensitive NTS neurons have been implicated in the regulation of breathing previously (Dean and Putnam, 2010). Nevertheless, it remains elusive regarding the neuronal phenotype. Accumulated in vitro studies demonstrated a depolarization or firing increase during physiological acidification in $\sim 35 \%$ of phenotype-unrevealed neurons (Dean et al., 1990), 40\% (acid-sensitive ion channel-containing) of neurons (Huda et al., 2012), or $43 \%$ of Phox 2 b-expressing neurons in the NTS (Fu et al., 2017). According to analysis of cell count, exposure to $5 \%-8 \% \mathrm{CO}_{2}$ produced activation of at least $12 \%$ of Phox $2 \mathrm{~b}$-expressing neurons in this transgenic mouse line. These neurons are, to a large extent, critical because they are presumed to provide an excitatory drive to rCPG by sensing excessive $\mathrm{CO}_{2} / \mathrm{H}^{+}$and, therefore, modulate a ventilatory response. Overall, our prior and present findings suggest that Phox $2 b-$ expressing NTS neurons are required for the hypercapnic ventilatory response. Nevertheless, our work is not sufficient to conclude that Phox $2 \mathrm{~b}$-expressing NTS neurons are important chemoreceptors. In other words, Phox $2 \mathrm{~b}$ does not seem a specific chemoreceptor signature in the NTS. A prior study has shown that $\mathrm{CO}_{2} / \mathrm{H}^{+}$-sensitive caudal NTS neurons responded to focal ATP application in brainstem slices; however, these chemosensitive neurons were Phox $2 b$ deficient (Sobrinho et al., 2014), raising the possibility that Phox2b-deficient NTS neurons probably function as respiratory chemoreceptors. In addition, we realize that destruction of any element of the respiratory circuit would likely diminish breathing and $\mathrm{CO}_{2}$ sensitivity, and this is consistent with the present evidence indicating that selective activation of Phox $2 \mathrm{~b}$ expressing NTS neurons increased cFos in the preBötC. Thus, future studies are necessary to identify the more specific marker of central chemoreceptor neurons or the subset of Phox $2 \mathrm{~b}$-expressing chemosensitive neurons in the NTS.

Interestingly, chemogenetic stimulation of Phox $2 b$-expressing neurons in the NTS synergized with moderate level of $\mathrm{CO}_{2}$ (5\%) exposure to enhance the ventilatory response, whereas no significant change in $\mathrm{MV}$ was found between $8 \% \mathrm{CO}_{2}$ alone and $8 \% \mathrm{CO}_{2}$ plus $\mathrm{CNO}$ stimulations. These effects were attributed to the following reasons. On one hand, in relative to systemic stimulation of $\mathrm{CO}_{2}$, activation of Phox2b-expressing NTS neurons has a limited capability for an enhancement of ventilatory responses. On the other hand, a number of Phox $2 \mathrm{~b}$-expressing NTS neurons could be recruited simultaneously by both $\mathrm{CO}_{2}$ and CNO stimulations; in particular, the number of shared Phox $2 \mathrm{~b}$ neurons is presumed to be greater in response to a higher level of $\mathrm{CO}_{2}(8 \%)$ despite paucity of direct evidence presented herein.

\section{Effect of Phox2b-expressing neuron stimulation on preBötC neuronal activity}

We presume that Phox $2 \mathrm{~b}$-expressing NTS neurons directly modulate rCPG neurons based on the following experiments. First, neuroanatomical evidence supported a direct projection of Phox $2 \mathrm{~b}$-expressing neurons to medullary ventrolateral respiratory column regions, including rVRG, preBötC, and BötC. Second, according to cFos-based analysis of cell count in the ventrolateral medulla, more preBötC neurons were activated after selective stimulation of Phox2b-expressing neurons. Third, activation of Phox $2 \mathrm{~b}$-expressing neurons markedly reduced both $\mathrm{T}_{\mathrm{i}}$ and $\mathrm{T}_{\mathrm{e}}$, which was probably ascribed to a regulatory effect of Phox $2 b$-expressing neuron activation on rCPG activity.

The notion is also consolidated by prior studies demonstrating extensive projections from NTS to the three serially arrayed regions (Geerling and Loewy, 2006; Rinaman, 2010; Alheid et al., 2011), whereas the phenotype of NTS projection neurons remains unknown. Nevertheless, the NTS neuron may influence rhythm-generating circuits in the preBötC via indirect links. Obvious candidates consist of projections from NTS to the parabrachial and Kölliker-Fuse nuclei (Song et al., 2011), rVRG (Moreira et al., 2007a), and RTN (Takakura et al., 2006, 2007; Moreira et al., 2007b), ultimately affecting the activity of preBötC neurons. The present study does not rule out the possibility that stimulation of Phox $2 \mathrm{~b}$-expressing neurons activated breathing via indirect links. 


\section{Translational implications and conclusion}

Here, chemogenetic stimulation of Phox2b-expressing NTS neurons produced a long-lasting potentiation of baseline pulmonary ventilation, identifying a neuronal subtype in the NTS for the control of breathing. The CCHS, mainly characterized by nocturnal sustained hypoventilation caused by mutant Phox $2 \mathrm{~b}$ (WeeseMayer et al., 2010), may be partially due to the loss (70\%) of RTN neurons (Dubreuil et al., 2008). Meanwhile, in the mouse model, Phox $2 \mathrm{~b}$-expressing neurons in the NTS were anatomically intact (Dubreuil et al., 2008), reminiscent of a notion that selective activation of Phox $2 b$-expressing NTS neurons by either molecular manipulation or pharmacological stimulation could improve hypoventilation presumably due to loss of RTN neurons.

In conclusion, selective stimulation of Phox $2 b$-expressing NTS neurons significantly potentiates baseline pulmonary ventilation. These neurons are also required for the hypercapnic ventilatory response. The circuit mechanism underlying the above effects is presumed to involve an excitatory drive of Phox $2 \mathrm{~b}$-expressing neurons to neuronal activity of rCPG. These findings would help us better understand neural circuits of respiratory control and present a clue to treatment of sleep-related hypoventilation or apnea.

\section{References}

Abbott SB, Stornetta RL, Fortuna MG, Depuy SD, West GH, Harris TE, Guyenet PG (2009) Photostimulation of retrotrapezoid nucleus Phox2b-expressing neurons in vivo produces long-lasting activation of breathing in rats. J Neurosci 29:5806-5819.

Alheid GF, Jiao W, McCrimmon DR (2011) Caudal nuclei of the rat nucleus of the solitary tract differentially innervate respiratory compartments within the ventrolateral medulla. Neuroscience 190:207-227.

Biancardi V, Bícego KC, Almeida MC, Gargaglioni LH (2008) Locus coeruleus noradrenergic neurons and $\mathrm{CO}_{2}$ drive to breathing. Pflugers Arch 455:1119-1128.

Dauger S, Pattyn A, Lofaso F, Gaultier C, Goridis C, Gallego J, Brunet JF (2003) Phox $2 \mathrm{~b}$ controls the development of peripheral chemoreceptors and afferent visceral pathways. Development 130:6635-6642.

Dean JB, Putnam RW (2010) The caudal solitary complex is a site of central $\mathrm{CO}_{2}$ chemoreception and integration of multiple systems that regulate expired $\mathrm{CO}_{2}$. Respir Physiol Neurobiol 173:274-287.

Dean JB, Bayliss DA, Erickson JT, Lawing WL, Millhorn DE (1990) Depolarization and stimulation of neurons in nucleus tractus solitarii by carbon dioxide does not require chemical synaptic input. Neuroscience 36:207-216.

Dubreuil V, Ramanantsoa N, Trochet D, Vaubourg V, Amiel J, Gallego J, Brunet JF, Goridis C (2008) A human mutation in Phox2b causes lack of $\mathrm{CO}_{2}$ chemosensitivity, fatal central apnea, and specific loss of parafacial neurons. Proc Natl Acad Sci U S A 105:1067-1072.

Dubreuil V, Barhanin J, Goridis C, Brunet JF (2009a) Breathing with Phox2b. Philos Trans R Soc Lond B Biol Sci 364:2477-2483.

Dubreuil V, Thoby-Brisson M, Rallu M, Persson K, Pattyn A, Birchmeier C, Brunet JF, Fortin G, Goridis C (2009b) Defective respiratory rhythmogenesis and loss of central chemosensitivity in Phox $2 \mathrm{~b}$ mutants targeting retrotrapezoid nucleus neurons. J Neurosci 29:14836-14846.

Fu C, Xue J, Wang R, Chen J, Ma L, Liu Y, Wang X, Guo F, Zhang Y, Zhang X, Wang S (2017) Chemosensitive Phox2b-expressing neurons are crucial for hypercapnic ventilatory response in the nucleus tractus solitarius. J Physiol 595:4973-4989.

Geerling JC, Loewy AD (2006) Aldosterone-sensitive neurons in the nucleus of the solitary tract: efferent projections. J Comp Neurol 497:223-250.

Guyenet PG (2006) The sympathetic control of blood pressure. Nat Rev Neurosci 7:335-346.

Huda R, Pollema-Mays SL, Chang Z, Alheid GF, McCrimmon DR, Martina M (2012) Acid-sensing ion channels contribute to chemosensitivity of breathing-related neurons of the nucleus of the solitary tract. J Physiol 590:4761-4775.

Kanbar R, Stornetta RL, Cash DR, Lewis SJ, Guyenet PG (2010) Photostimulation of Phox $2 \mathrm{~b}$ medullary neurons activates cardiorespiratory function in conscious rats. Am J Respir Crit Care Med 182:1184-1194.

Kumar NN, Velic A, Soliz J, Shi Y, Li K, Wang S, Weaver JL, Sen J, Abbott SB,
Lazarenko RM, Ludwig MG, Perez-Reyes E, Mohebbi N, Bettoni C, Gassmann M, Suply T, Seuwen K, Guyenet PG, Wagner CA, Bayliss DA (2015) Regulation of breathing by $\mathrm{CO}_{2}$ requires the proton-activated receptor GPR4 in retrotrapezoid nucleus neurons. Science 348:1255-1260.

Lazarenko RM, Milner TA, Depuy SD, Stornetta RL, West GH, Kievits JA, Bayliss DA, Guyenet PG (2009) Acid sensitivity and ultrastructure of the retrotrapezoid nucleus in Phox2b-EGFP transgenic mice. J Comp Neurol 517:69-86.

Li A, Nattie E (2006) Catecholamine neurones in rats modulate sleep, breathing, central chemoreception and breathing variability. J Physiol 570:385-396.

Moreira TS, Takakura AC, Colombari E, Guyenet PG (2007a) Activation of 5-hydroxytryptamine type 3 receptor-expressing C-fiber vagal afferents inhibits retrotrapezoid nucleus chemoreceptors in rats. J Neurophysiol 98:3627-3637.

Moreira TS, Takakura AC, Colombari E, West GH, Guyenet PG (2007b) Inhibitory input from slowly adapting lung stretch receptors to retrotrapezoid nucleus chemoreceptors. J Physiol 580:285-300.

Nattie EE, $\mathrm{Li} \mathrm{A}$ (2002) $\mathrm{CO}_{2}$ dialysis in nucleus tractus solitarius region of rat increases ventilation in sleep and wakefulness. J Appl Physiol 92:2119-2130.

Pattyn A, Morin X, Cremer H, Goridis C, Brunet JF (1999) The homeobox gene Phox $2 \mathrm{~b}$ is essential for the development of autonomic neural crest derivatives. Nature 399:366-370.

Paxinos G, Franklin KB (2003) The mouse brain in stereotaxic coordinates, Ed 2. San Diego: Academic.

Resch JM, Fenselau H, Madara JC, Wu C, Campbell JN, Lyubetskaya A, Dawes BA, Tsai LT, Li MM, Livneh Y, Ke Q, Kang PM, Fejes-Tóth G, Náray-Fejes-Tóth A, Geerling JC, Lowell BB (2017) Aldosteronesensing neurons in the NTS exhibit state-dependent pacemaker activity and drive sodium appetite via synergy with angiotensin II signaling. Neuron 96:190-206.e7.

Rinaman L (2010) Ascending projections from the caudal visceral nucleus of the solitary tract to brain regions involved in food intake and energy expenditure. Brain Res 1350:18-34.

Scott MM, Williams KW, Rossi J, Lee CE, Elmquist JK (2011) Leptin receptor expression in hindbrain glp-1 neurons regulates food intake and energy balance in mice. J Clin Invest 121:2413-2421.

Sobrinho CR, Wenker IC, Poss EM, Takakura AC, Moreira TS, Mulkey DK (2014) Purinergic signaling contributes to chemoreception in the retrotrapezoid nucleus but not the nucleus of the solitary tract or medullary raphe. J Physiol 592:1309-1323.

Song G, Xu H, Wang H, Macdonald SM, Poon CS (2011) Hypoxia-excited neurons in NTS send axonal projections to Kölliker-Fuse/parabrachial complex in dorsolateral pons. Neuroscience 175:145-153.

Takakura AC, Moreira TS, Colombari E, West GH, Stornetta RL, Guyenet PG (2006) Peripheral chemoreceptor inputs to retrotrapezoid nucleus (RTN) $\mathrm{CO}_{2}$-sensitive neurons in rats. J Physiol 572:503-523.

Takakura AC, Moreira TS, West GH, Gwilt JM, Colombari E, Stornetta RL, Guyenet PG (2007) GABAergic pump cells of solitary tract nucleus innervate retrotrapezoid nucleus chemoreceptors. J Neurophysiol 98:374-381.

Takakura AC, Moreira TS, Stornetta RL, West GH, Gwilt JM, Guyenet PG (2008) Selective lesion of retrotrapezoid Phox2b-expressing neurons raises the apnoeic threshold in rats. J Physiol 586:2975-2991.

Tiveron MC, Pattyn A, Hirsch MR, Brunet JF (2003) Role of Phox2b and Mash1 in the generation of the vestibular efferent nucleus. Dev Biol 260:46-57.

Wang S, Shi Y, Shu S, Guyenet PG, Bayliss DA (2013a) Phox2b-expressing retrotrapezoid neurons are intrinsically responsive to $\mathrm{H}^{+}$and $\mathrm{CO}_{2}$. J Neurosci 33:7756-7761.

Wang S, Benamer N, Zanella S, Kumar NN, Shi Y, Bévengut M, Penton D, Guyenet PG, Lesage F, Gestreau C, Barhanin J, Bayliss DA (2013b) TASK-2 channels contribute to $\mathrm{pH}$ sensitivity of retrotrapezoid nucleus chemoreceptor neurons. J Neurosci 33:16033-16044.

Weese-Mayer DE, Berry-Kravis EM, Ceccherini I, Keens TG, Loghmanee DA, Trang H (2010) An official ATS clinical policy statement: congenital central hypoventilation syndrome: genetic basis, diagnosis, and management. Am J Respir Crit Care Med 181:626-644.

Zhao Z, Wang L, Gao W, Hu F, Zhang J, Ren Y, Lin R, Feng Q, Cheng M, Ju D, Chi Q, Wang D, Song S, Luo M, Zhan C (2017) A central catecholaminergic circuit controls blood glucose levels during stress. Neuron 95:138-152.e5. 